\title{
Meta
}

Journal des traducteurs

Translators' Journal

\section{Gile, Daniel (1995) : Basic Concepts and Models for Interpreter and Translator Training, Amsterdam/Philadelphia, John Benjamins, 274 p.}

\section{Louise Brunette}

Volume 43, numéro 2, juin 1998

URI : https://id.erudit.org/iderudit/002162ar

DOI : https://doi.org/10.7202/002162ar

Aller au sommaire du numéro

Éditeur(s)

Les Presses de l'Université de Montréal

ISSN

0026-0452 (imprimé)

1492-1421 (numérique)

Découvrir la revue

Citer ce compte rendu

Brunette, L. (1998). Compte rendu de [Gile, Daniel (1995) : Basic Concepts and Models for Interpreter and Translator Training, Amsterdam/Philadelphia, John Benjamins, 274 p.] Meta, 43(2), 320-325. https://doi.org/10.7202/002162ar d'utilisation que vous pouvez consulter en ligne.

https://apropos.erudit.org/fr/usagers/politique-dutilisation/ 


\section{DOCUMENTATION}

\section{Comptes rendus}

- GILE, Daniel (1995) : Basic Concepts and Models for Interpreter and Translator Training, Amsterdam/Philadelphia, John Benjamins, 274 p.

Pour les traductologues en général, mais particulièrement pour les pédagogues de la traduction et de l'interprétation, l'annonce d'un nouveau titre de Daniel Gile a toujours des allures de promesse. Basic Concepts and Models for Interpreter and Translator Training désormais abrégé en Basic Concepts — était attendu. L'attente n'a pas été déçue, puisqu'on se trouve en présence d'un ouvrage que sans doute nombre de didacticiens de la traduction/ interprétation voudraient avoir écrit.

Pour n'être pas toutes nouvelles, les idées exprimées dans Basic Concepts n'en demeurent pas moins originales, en ce sens qu'elles reflètent bien l'orientation et la didactique propres à l'auteur au chapitre des discours non littéraires. En fait, le livre rassemble, parfois en les mettant à jour et en les enrichissant, les réflexions et les prises de position présentées par Daniel Gile dans ses articles et autres publications antérieures, même les plus récentes. Basic Concepts peut se définir à la fois comme monographie et comme manuel de pédagogie. Monographie, il fait le point sur l'état actuel de la traductologie et de la traduction/interprétation; manuel de pédagogie, il éclaire l'enseignant chargé lui-même d'orienter l'étudiant dans l'acquisition des habiletés et des connaissances devant en faire un bon traducteur/interprète. Les thèmes traités illustrent bien cette orientation hybride puisque le lecteur naviguera entre des descriptions de modèles théoriques, des démarches pédagogiques sous forme de stratégies à appliquer en classe de traduction/interprétation, des aspects concrets de l'exercice comme l'interprétation à vue, ou empiriques comme la fatigue de l'interprète; Gile attire même l'attention sur des éléments psychologiques, la fierté de l'interprète, par exemple. Mais il nous convie à une visite guidée et, compte tenu de l'ampleur du sujet traité, on peut considérer comme un tour de force l'unité de l'ensemble de son livre.

Dans une langue toujours simple et efficace, jamais jargonnée, Daniel Gile a composé dix chapitres équilibrés du point de vue du contenu et de la longueur et presque tous construits selon le même plan : exposé général, applications pédagogiques, synthèse. Le premier chapitre détermine la double clientèle et les objectifs du livre. Double clientèle, car si les lecteurs sont des enseignants, il ne faut pas oublier leurs étudiants : les futurs traducteurs et interprètes dont la formation devrait reposer davantage sur la présentation des stratégies traductionnelles que sur la mise en évidence des erreurs. C'est pourquoi Gile accorde une place privilégiée aux principes et aux modèles, sans jamais toutefois négliger la pratique. Il écrit d'ailleurs : «Prior to translation exercices, methodological guidance is given in the form of

Meta, XLIII, 2, 1998 
basic concepts and models. This book is essentially a presentation of such concepts and models.» (p. 10). Le pédagogue distingue aussi deux types de cursus : des cours destinés à des néophytes et des programmes s'adressant aux praticiens désireux de parfaire leurs connaissances et leur pratique. Dans les deux cas, l'enseignement de la théorie est valable dans la mesure où il donne aux étudiants accès à des stratégies concrètes de traduction/interprétation, dans la mesure aussi où le contenu théorique des cours reste en prise sur la réalité quotidienne du praticien. Le chapitre se termine sur la description et la justification des modèles annoncés et prônés, qui font figure d'objectifs pédagogiques et concernent plus spécifiquement la traduction/interprétation comme acte de communication, le traitement de l'information, la compréhension des discours spécialisés, les étapes de l'acte traductif, la série d'efforts inhérents à la prestation de traduction/interprétation et la disponibilité du bagage linguistique de chaque traducteur/interprète.

La qualité constitue le thème du chapitre suivant. L'auteur établit ici une différence entre deux notions parfois confondues : la traduction pédagogique, axée sur le linguistique, et la traduction professionnelle, acte fonctionnel de communication. Chez Daniel Gile, le phare de la traduction est la finalité du texte. En fait, dans le contexte pédagogique, la qualité se définirait toujours par rapport à l'opération de communication (par opposition à des objectifs politiques, administratifs, etc.) même si, dans la pratique, d'autres facteurs, commerciaux notamment, interviennent. Par ailleurs, Gile démontre combien le contenu du concept de qualité varie suivant l'agent en cause. Mentionnons que tous les principes examinés dans le chapitre 2 sont facilement assimilables par des profanes, par des débutants. Par ailleurs, la notion de qualité vue par Gile s'impose par son caractère pratique, empirique. La réalité qui attend les étudiants est bien la suivante : en traduction/interprétation, les jugements non critériés sur les prestations dépassent largement en nombre les révisions ou les évaluations systématiques appliquant des grilles éprouvées : «[...] the actual assessment of quality depends inter alia on the specific needs of the assessor, and [...] overall quality assessment can vary significanlty in any given context for this reason.» (p. 38).

À cause des multiples réalités qu'on lui a fait recouvrir, le terme «fidélité» a perdu aujourd'hui, traductologiquement, tout sens précis. Mais si le concept s'est embrouillé, l'étiquette reste populaire, et la fidélité est le thème du chapitre 3 consacré à la transmission satisfaisante de l'information à faire passer. Le lecteur prend connaissance d'un véritable modèle, assimilable à un plan de cours intégrant la gradation des difficultés, bien qu'il ne soit pas fait expressément mention de cette gradation dans le texte de Gile. Conformément à la mission et au projet qu'il s'est donnés, l'auteur indique aux formateurs à quel point du cursus la notion de fidélité doit s'insérer : «[...] the informational model of informative sentences should be introduced at a very early stage in the syllabus, preferably immediately after the modules on communication and quality.» (p. 50).

Pour illustrer ses propos sur la fidélité, Daniel Gile se sert d'expériences menées sur des groupes choisis, dont l'une est inspirée par le souci d'éliminer chez les étudiants le réflexe du mot à mot. Ainsi rapporte-t-il un travail sur les multiples façons (signes graphiques) d'exprimer un même message à partir de la même mise en situation. La méthode d'enquête et les résultats convainquent. Par exemple, l'expérience a été reprise avec des échantillons (quelque 1000 répondants) différents quant à l'origine et à la langue maternelle. Si l'auteur ne met pas à la disposition du lecteur des données à proprement parler recueillies sur le terrain, c'est-à-dire collectées auprès de professionnels en exercice, la qualité de l'échantillonnage (nombre et types de traducteurs) compense largement l'artificialité du laboratoire. Par ailleurs, la compilation de Gile met en lumière l'importance de la liberté dans l'expression et la parfaite compatibilité de cette liberté et de la transmission fidèle de l'information. Les conclusions de l'exercice soulignent aussi la tendance des traducteurs débutants ou expérimentés à vouloir TOUT réexprimer de façon à ne rien manquer. 
Sur le plan théorique, Gile affirme que sur un tronc commun d'information, ou nœud du message, viennent se greffer des données secondaires, réparties en trois catégories, selon leur source : la situation (Framing Information), les renseignements fournis par la «grammaire» (Linguistically Induced Information (ex. : nombre de personnes en cause)) et les éléments à relier à l'expérience du locuteur original (Personal Information) et déterminés par son bagage émotif ou cognitif, sa classe sociale même. En matière de fidélité, d'après l'auteur, les traducteurs s'entendent sur l'obligation absolue de reporter dans la traduction l'information de base (kernel) du texte lu ou entendu. Pour les autres facteurs informatifs, il ne se dégage pas de règle fixe. Il revient au traducteur/interprète de faire ses choix, de prendre ses décisions, mais en se méfiant des explicitations indues, révélatrices d'une méthode déficiente et sources de foisonnement.

Bien sûr, ces principes ont leurs retombées pédagogiques : on enseignera que si le «message» est «sacré», des ajouts et des suppressions restent possibles en matière d'informations secondaires. Il faut toutefois veiller, précisera-t-on aux étudiants, à procéder dans le respect de la hiérarchie suivante : situation, éléments reliés à la personnalité de l'émetteur et formes linguistiques.

Le chapitre 4, articulé autour du troisième modèle, se résume à l'analyse des différents constituants de la compréhension, mais dans la seule perspective pédagogique, et sans entrer dans les détails. L'accent est mis sur deux facteurs, le linguistique et l'extralinguistique, et sur leur interaction complémentaire et synergique. Pour Gile, la compréhension peut supposer et supporter des degrés. En fait, il est tout à fait concevable et acceptable que le traducteur/interprète comprenne plus ou moins les signes graphiques ou phoniques enregistrés. En traduction, il importe d'arriver à déterminer le degré de compréhension nécessaire à la reformulation honnête du message. À cette fin, l'auteur indique des balises (p. 82) :

In non-literary interpretation and translation, the need to reformulate the information content of the original Text in the target language means that its logical and functional infrastructure [au singulier] must be understood to the point where it can be disambiguated enough to be meaningfully reworded in the target language and where the appropriate terms in the target language can be selected by the Translator, if they exist, or be created, if they do not.

Comme il a déjà été mentionné, les textes et discours auxquels s'applique le modèle de compréhension de Gile appartiennent aux domaines spécialisés. Coiffant de nouveau son chapeau de formateur, l'auteur rappelle aux lecteurs des règles connues dans l'enseignement de la traduction technique. Ainsi, dans certains cas, le traducteur spécialisé aura intérêt, pour aiguiser sa compréhension, à parfaire sa formation dans un domaine de spécialité plutôt qu'en linguistique ou en littérature. Il sera ainsi en mesure de procéder relativement facilement à l'analyse logique des textes spécialisés. Par ailleurs, de l'avis de Gile, même en traduction/interprétation technique ou spécialisée, on prendra garde d'exagérer la profondeur des connaissances à acquérir avant de commencer à traduire, puisque la logique vient souvent à la rescousse du traducteur. Sur le plan didactique, cette insistance sur l'analyse, premier outil du traducteur/interprète, révèle aux enseignés combien la saisie de textes techniques est plus à leur portée qu'ils ne l'auraient imaginé. À une étape ultérieure, souligne Gile, on intégrera à cette compréhension prenant appui sur la logique, les contraintes stylistiques et terminologiques. En outre, le pédagogue ne manque pas de signaler combien la situation se complique lorsque le traducteur n'arrive pas à rétablir le fil de la pensée, par exemple s'il doit composer avec le style télégraphique, caractéristique notamment des textes sur diapositives.

Au chapitre 5, Daniel Gile expose son modèle séquentiel ou, plus simplement, ce qu'il considère être les étapes de l'acte de traduction/interprétation. Il s'agit pour l'auteur de tracer à l'intention du futur professionnel le cheminement conduisant du discours de départ au dis- 
cours traduit. Loin de le poser comme une démarche incontournable, Daniel Gile propose le modèle séquentiel — aujourd'hui presque universellement accepté — comme un possible outil pédagogique à présenter éventuellement aux futurs traducteurs dès après leur initiation aux notions plus ou moins théoriques de qualité, de fidélité et de compréhension.

Quelles sont ces étapes ? Pour chaque unité de traduction, une hypothèse de sens est posée. Puis, la plausibilité de l'hypothèse est vérifiée jusqu'à l'émergence d'une analyse satisfaisante et acceptable. Suit la formulation. Dans les cas de traductions écrites, on entre alors dans une phase de contrôle concernant notamment l'intégrité du message, la justesse $\mathrm{du}$ vocabulaire, la correction linguistique. Le traducteur relit son texte au complet pour en vérifier la cohérence et la cohésion. La méthode serait applicable à la production de n'importe quel texte (traduction ou original).

Pour que se vérifie la pertinence du modèle séquentiel, Daniel Gile le précise, il faut, avec les débutants, n'utiliser que des textes compréhensibles pour ces derniers. Les textes qui ne remplissent pas cette condition n'auront aucune chance de se rendre à l'étape de la reformulation sans compter qu'ils ne laisseront pas aux apprenants la chance d'avancer des hypothèses. Or, un des buts visés par la méthode est de placer les étudiants devant une des réalités de leur future profession : traduire, c'est prendre des décisions.

Acquérir des connaissances en anglais et en français ou comment se préparer à traduire un texte ou à interpréter un discours. Le chapitre 6 de Basic Concepts pourrait bien s'intituler ainsi en français. Ses 25 pages comportent des renseignements pratiques sur la démarche documentaire, clairement décrite. Une fois établie la nécessité pour le traducteur d'avoir à sa disposition des sources terminologiques (les plus efficaces, semble-t-il, pour les interprètes, en particulier) ou techniques dignes de foi, Gile fait un tour d'horizon de l'information disponible, en insistant sur le caractère inévitablement obsolète ou confidentiel de toutes les publications techniques ou para-techniques d'aujourd'hui et, conséquemment, en mettant l'accent sur la place privilégiée des sources humaines.

On trouve dans ces pages des conseils judicieux sur l'initiation à la documentation des traducteurs et des interprètes apprentis, par exemple sur l'importance de leur inculquer les critères d'évaluation de la documentation. Conformément à sa ligne de conduite, l'auteurpédagogue indique aux lecteurs enseignants le moment opportun pour procéder à l'insertion de cette matière dans le cursus.

Avec le chapitre 7, Gile aborde les modèles d'effort. Se trouve ainsi mise en lumière une vérité qui pourrait passer pour une banalité mais à laquelle mieux vaut sensibiliser le plus tôt possible les aspirants traducteurs/interprètes : traduire requiert du talent et de l'énergie; pour bien traduire, il faut s'y préparer et consentir à des efforts soutenus. Gile ne le cache pas : le métier d'interprète est difficile, fatigant. Ainsi, interpréter exige une attention constante en même temps que cet exercice prive l'interprète de sa spontanéité, puisque la performance de ce dernier ne peut s'appuyer sur des automatismes, langagiers par exemple. L'interprète se trouve constamment sollicité : il doit comprendre, analyser, mémoriser, prendre des notes (en consécutive), éviter les interférences (particulièrement en traduction à vue) et reformuler le discours tout à la fois. Que l'énergie spécifique vienne à lui manquer pour exercer une de ces fonctions, et son rendement en souffre. Une des différences entre le traducteur et l'interprète tiendrait précisément à ce «multitâche» qui oblige l'interprète à gérer tous ses efforts simultanément.

Comme le précédent, le chapitre 8 concerne presque essentiellement l'interprétation. Il prend la forme d'une énumération, explicative, de stratégies curatives ou préventives et de leurs modes d'emploi respectifs, face à des problèmes particuliers en interprétation, consécutive ou simultanée : difficultés de compréhension du discours (communication), difficultés de réexpression. Les solutions décrites ont parfois un caractère méthodologique comme, par exemple, l'utilisation du procédé (dangereux) de calque; elles prennent parfois des allu- 
res très pragmatiques comme dans les cas d'utilisation de documentation ou du recours au collègue en cabine ou à l'orateur lui-même. Toutefois, il ne saurait ici être question de «recettes» au sens péjoratif du terme, car Gile refuse catégoriquement toute stratégie inspirée par la loi du moindre effort (p. 203) : «The Law of least effort [...] should [...] be considered an unwelcome intruder, which entails loss of information without good reason.» D'ailleurs, le respect de l'éthique teinte tout le livre. Concret, Gile décrit aussi les moyens à la disposition des interprètes pour contrer, notamment, les malaises issus des erreurs imputables aux orateurs. Pédagogiquement parlant, Gile nous indique encore comment exposer les stratégies en cause aux étudiants, à quelle étape du cursus le faire et dans quel environnement.

L'avant-dernier chapitre s'ouvre sur les définitions théoriques et classiques des langues dites respectivement A, B et C. Suivent des constatations aussi déprimantes qu'avérées : la plupart des écoles de traduction acceptent des candidats dont la compétence en langue ne correspond pas aux exigences du métier qu'ils ont choisi, et ces établissements doivent un jour ou l'autre incorporer des cours de perfectionnement linguistique dans des programmes conçus avec d'autres objectifs. Faisant contre mauvaise fortune bon cœur et pour faire assimiler ces compétences, Gile livre à la réflexion des didacticiens un nouveau modèle représenté par des cercles concentriques gravitant autour d'un noyau et fondé sur le principe de l'accès plus ou moins immédiat pour l'interprète — il est peu question des traducteurs dans le chapitre 9 - à son bagage lexical, syntaxique et grammatical. Certains éléments de base n'ont pour ainsi dire jamais besoin d'être réactivés; c'est le contraire pour les autres. Pour comprendre et simplifier le modèle, disons que le lecteur peut se rabattre sur les notions habituelles de vocabulaire passif et de vocabulaire actif en y ajoutant les interactions ou migrations entre les deux. Gile explique ce modèle par des règles dont la description souligne particulièrement l'importance des interférences linguistiques et les différences entre la nature et les conséquences de ce phénomène de contact de langues selon que l'on pratique l'interprétation ou la traduction. L'auteur propose ici aussi des applications pédagogiques de son modèle, le dernier, et il en dégage les implications didactiques. En matière de stratégies d'acquisition du vocabulaire pour les interprètes en formation ou professionnels, le modèle conduit l'auteur à suggérer concurremment aux méthodes scolaires (ex. : choix des conférences à faire interpréter), différents types d'immersions : géographiques, électroniques (lectures) et culturelles. Cette incursion du côté de la langue donne lieu à une réhabilitation du caractère linguistique de l'opération traduisante qui termine le chapitre.

Tout le dernier chapitre, le dixième, est occupé par une bibliographie sélective, commentée, touchant plus ou moins directement la formation des interprètes et des traducteurs. Par exemple, en ce qui concerne les publications ad hoc, l'auteur dresse pour les enseignants et leurs étudiants une liste de périodiques savants ou professionnels; il fournit aussi des informations sur les thèses et mémoires, sur des monographies, des actes, des publications internes d'organismes divers et, surtout, il évalue la documentation décrite.

Une place relativement importante revient aux publications des écoles de traduction avec ce qui semble au premier abord, une répartition équitable entre les pays de l'Est et ceux de l'Ouest. Sont aussi mentionnés des collectifs produits par des regroupements d'interprètes ou de traducteurs : AIIC, FIT, AILA, ATA. Daniel Gile établit ensuite une typologie originale des auteurs dans les domaines qui intéressent la traductologie : praticiens, péda-traducteurs ou interprètes, chercheurs universitaires ou chercheurs autonomes, chercheurs à statut mixte. Renonçant à la neutralité qui marque l'ensemble de Basic Concepts, il s'en prend assez durement aux chercheurs autonomes (Developing Researchers) dont il fustige l'ignorance des principes scientifiques de la recherche. 
Par ailleurs, conscient que toutes les bonnes idées en matière d'enseignement ne sont pas consignées dans les revues professionnelles ou savantes, l'auteur encourage les formateurs à s'alimenter aussi aux discussions avec leurs collègues et confrères.

Daniel Gile propose aussi la lecture d'ouvrages de base et celle de certains auteurs en psychologie cognitive ou en psycholinguistique. Finalement, il passe en revue quinze publications, des classiques pour la plupart, en français ou en anglais qui devraient renseigner les enseignants sur les orientations contemporaines, théoriques et pratiques, de la didactique de la traduction.

Enfin, il faut souligner la richesse de la bibliographie générale, qui couvre au moins quatre sujets : pédagogie, terminologie, traduction, interprétation et concerne trois langues : l'anglais, le français et le japonais.

L'ouvrage de Daniel Gile suscite davantage de commentaires qu'il ne s'attire de critiques. Cependant, le lecteur pédagogue pourra ne pas donner son aval à toutes les démarches recommandées par l'auteur. Par exemple, on pourrait contester la pertinence de certaines méthodes de vérification plus près de la postédition que de la traduction : «[...] to start with a word-for-word translation and then work one's way up toward acceptable wording through the reformulation loop.» (p. 117). Certains aussi, s'opposant à Gile, ne recommanderont jamais à leurs étudiants ou à leurs révisés de s'en remettre au mot à mot pour tenter de sortir d'une difficulté de compréhension (p. 124).

Ces objections mises à part, restent les commentaires, aussi d'ordre didactique. Ainsi, il aurait été éclairant de savoir sur combien de trimestres Gile administre l'enseignement décrit dans Basic Concepts; de même on aurait apprécié un peu plus de précision sur les répercussions de l'enseignement de la théorie dans les cours de traduction/interprétation; finalement, toujours en matière de pédagogie, Gile ne nous fait pas voir assez distinctement la différence, soulignée dans les premières pages, entre les cursus des traducteurs néophytes et ceux des praticiens en quête de perfectionnement.

Sur le fond, un seul sujet d'agacement: l'absence de critères précis sur l'évaluation qualitative des traductions. Ce silence étonne un peu dans un ouvrage où la qualité fait l'objet de tout un chapitre. Mais on le pardonnera à l'auteur pour retenir surtout que, solidement ancré dans la pratique tout en reposant sur des assises théoriques solides, Basic Concepts devrait marquer la pédagogie de la traduction. En effet, Daniel Gile est peut-être le premier à livrer au monde de la traduction/interprétation une monographie atteignant avec le même bonheur formateurs et étudiants. C'est pourquoi la didactique de la traduction devra dorénavant compter avec Basic Concepts.

LOUISE BRUNETTE

Université Concordia, Montréal, Canada 